\title{
Fast Image Decoding for Block Compressed Sensing based encoding by using a Modified Smooth $l_{0}$-norm
}

\author{
Xiao Jieqiong
}

\author{
Carlos R. del-Blanco, Carlos Cuevas, Narciso García
}

\begin{abstract}
This paper proposes a fast decoding algorithm for block-based compressed sensing images that combines a modified smooth $l_{0}$-norm with the BCS-SPL algorithm. Experimental results have proven a significant reduction in execution time, while providing the same image quality.
\end{abstract}

Keywords - compressed sensing; SL0; BCS-SPL; modified Newton method

\section{INTRODUCTION}

Compressed sensing is a novel sampling paradigm that reduces the sampling rate imposed by the traditional Nyquist/Shannon sampling theorem for signals that are sparse or compressible in some domain [1]. The sampling process of Compressive Sensing could be utilized in many consumer electronics (CE) applications dealing with sound, image, and video signals. This fact has called the attention of many researchers in the field of image compression. For example, the BCS algorithm [2] proposes a block compressed sensing algorithm that divides the original image into small blocks, and then uses the same measurement matrix for all of them, achieving a great memory reduction. However, it suffers from obvious blocking artifacts due to the block operation. To mitigate these blocking artifacts, the BCS-SPL algorithm [3] combines Wiener filter with the Projected Landweber technique. But, the iterative-based reconstruction process is slow due to the poor convergence speed under low sampling rates. In addition, the reconstructed images tends to be blurred.

This paper proposes to use a modified smooth $l_{0}$-norm (SL0) within the formulation of the BCS-SPL algorithm to reduce the iteration time, achieving a better execution time performance. This new algorithm is referred to as BCS-NPLSL0.

\section{BACKGROUND}

The SL0 algorithm [4] uses a smoothing Gaussian function to approximate the $l_{0}$-norm. This allows it to both obtain the minimum value and reduce the sensitivity to noise. The problem of finding the sparsest solution is cast to solve a constrained minimization problem. SLO iteratively uses steepest descent method to solve the previous minimization problem, diminishing the step magnitude in every iteration to guarantee that the $l_{0}$-norm approximation function is smooth, which in turn avoids getting trapped into local minimum. However, an aliasing effect is created due to its negative iteration direction, complicating the calculation of the iteration step. To overcome this shortcoming, Lin proposed the NSL0 algorithm [5], which uses hyperbolic tangent function:

$$
f_{\sigma}\left(x_{i}\right)=\frac{e^{\frac{x_{i}^{2}}{2^{\sigma^{2}}}}-e^{\frac{x_{i}^{2}}{2^{\sigma^{2}}}}}{e^{\frac{x_{i}^{2}}{2^{\sigma^{2}}}}+e^{-\frac{x_{i}^{2}}{2^{\sigma^{2}}}}}
$$

to approximate the $l_{0}$-norm, where $\sigma$ is a parameter and $x_{i}$ is the $\mathrm{i}$-th component of the original signal $x$.

Instead of using Gaussian function

$$
\varphi_{\sigma}\left(x_{i}\right)=\exp \left(-\frac{x_{i}^{2}}{2 \sigma^{2}}\right)
$$

The hyperbolic tangent function and the Gaussian function are related by:

$$
f_{\sigma}\left(x_{i}\right) \geq 1-\varphi_{\sigma}\left(x_{i}\right)
$$

We define:

$$
F_{\sigma}(x)=\sum_{i=1}^{N} f_{\sigma}\left(x_{i}\right)
$$

When $\sigma \rightarrow 0$, the value of $F_{\sigma}(x)$ will approximate the number of non-zero elements in $x$. The $l_{0}$-norm of original signal $x$ can be represented by:

$$
\|x\|_{0}=\lim _{\sigma \rightarrow 0} F_{\sigma}(x)
$$




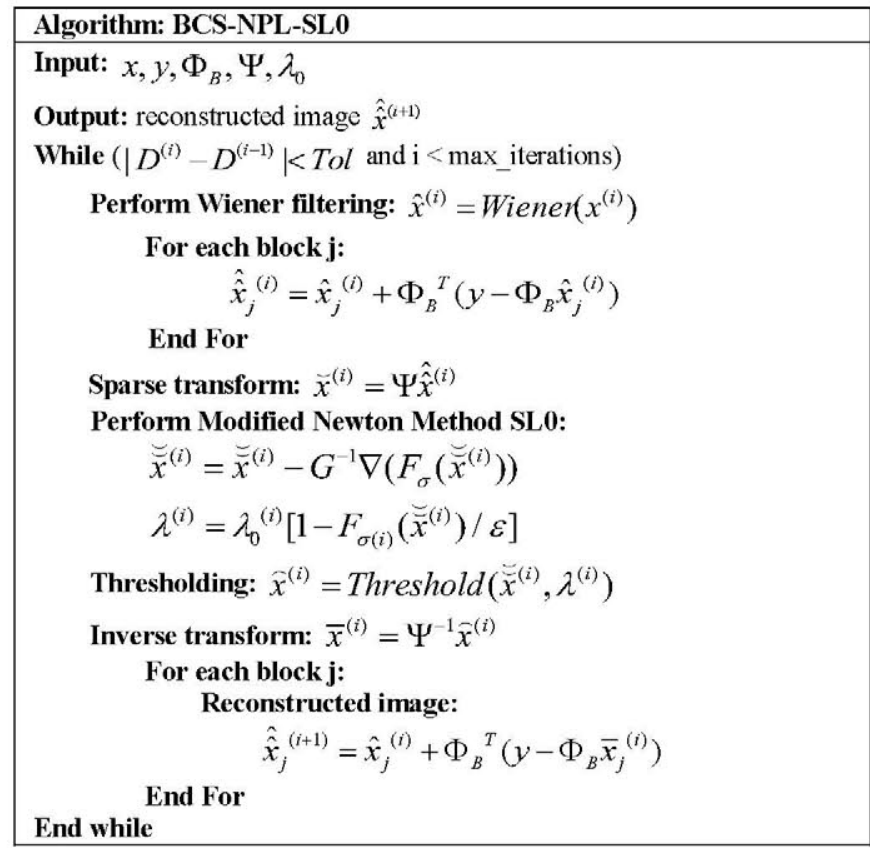

Fig. 1. Proposed CS recovery algorithm BCS-NPL-SL0

Therefore, for all the $\sigma \geq 0$, choosing the hyperbolic tangent function to approximate the $l_{0}$-norm can result in better convergence than choosing Gaussian function. Meanwhile, the NSL0 algorithm improves the convergence speed by using the modified Newton method to replace the steepest descent method.

This paper proposes combining the advantages of both NSLO and BCS-SPL algorithms to significantly improve the computational time of the reconstruction step involved in blocked compressed sensing images. First, NSL0 uses the modified Newton method to approximate global solution [5], achieving a fast convergence, and a shorter reconstruction time. And then, it is combined with BCS-SPL to reduce the iteration time under conditions of low sampling rate. As a result, a high quality reconstructed block-based Compressed Sensing image is fastly obtained.

\section{PROPOSED BCS-NPL-SL0 METHOD}

The SL0 algorithm is applied to a modified version of the Newton method, which is then iteratively combined with the BCS-SPL technique to achieve the best reconstructed blockbased Compressed Sensing image.

For the i-th iteration, Wiener filtering with a neighborhood of $3^{*} 3$ processes the original image $x^{(i)}$ to obtain $\hat{x}^{(i)}$. Then $\hat{x}^{(i)}$ is divided into blocks, where the $\mathrm{j}$-th block of the $\mathrm{i}$-th iteration is represented as $x_{j}^{(i)}$. The reconstruction error is then decreased in each iteration by $\hat{\hat{x}}_{j}^{(i)}=\hat{x}_{j}^{(i)}+\Phi_{B}{ }^{T}\left(y-\Phi_{B} \hat{x}_{j}^{(i)}\right)$, where $\Phi_{B}$ is an orthonormalized i.i.d Gaussian matrix [2]. Then we project the whole image $\hat{\hat{x}}^{(i)}$ to the sparse domain to get $\breve{x}^{(i)}$. Next, the Modified Newton Method is combined with SL0 to improve the convergence speed. We calculate the Newton direction of the hyperbolic tangent function. To guarantee the Hessian matrix in the calculation is positivedefinite, we modify the Hessian matrix to obtain a fixed Newton direction:

$$
d=-G^{-1} \nabla\left(F_{\sigma}\left(x^{(i)}\right)=\left[-\frac{\sigma^{2} x_{1}^{(i)}}{\sigma^{2}+\left(x_{1}^{(i)}\right)^{2}}, \ldots,-\frac{\sigma^{2} x_{n}^{(i)}}{\sigma^{2}+\left(x_{n}^{(i)}\right)^{2}}\right]^{T}\right.
$$

Then we perform the minimum approximation: $\breve{x}^{(i)}=\breve{x}^{(i)}+d$. At the same time, we adjust the threshold shrinkage coefficient $\lambda^{(i)}$ accordingly: $\lambda^{(i)}=\lambda_{0}^{(i)}\left[1-F_{\sigma(i)}\left(\breve{x}^{(i)}\right) / \varepsilon\right]$ to manage the convergence speed. Then, the hard thresholding [2] is performed to the input signal: $\tilde{x}^{(i)}=\operatorname{Threshold}\left(\widetilde{x}^{(i)}, \lambda^{(i)}\right)$ and project the sparse signal back to the original domain with the inverse transform $\Psi^{-1}: \bar{x}^{(i)}=\Psi^{-1} \widehat{x}^{(i)}$. Thus, the approximation to the image block $\mathrm{j}$ at the $(\mathrm{i}+1)$-th iteration is: $\hat{\hat{x}}_{j}^{(i+1)}=\bar{x}_{j}^{(i)}+\Phi^{T}\left(y-\Phi \bar{x}_{j}^{(i)}\right)$.

The iteration stops when $\left|D^{(i)}-D^{(i-1)}\right|<T o l$, where $D^{(i)}=\left\|x^{(i)}-\hat{\hat{x}}^{(i-1)}\right\|_{2}$, and Tol is a tolerance value. At each iteration, the SL0 method helps the algorithm to achieve a faster convergence, whereas a modified Newton method finds the global minimum, avoiding local minima solutions. Fig. 1 describes step-by-step the proposed algorithm.

\section{EXPERIMENTAL RESULTS}

The proposed BCS-NPL-SL0 algorithm has been compared with the BCS-SPL algorithm using three commonly used images: Lenna, Barbara, and Peppers. The subrate is set from 0.1 to 0.9 , and the block size is $32 * 32$. Parameters for BCS-

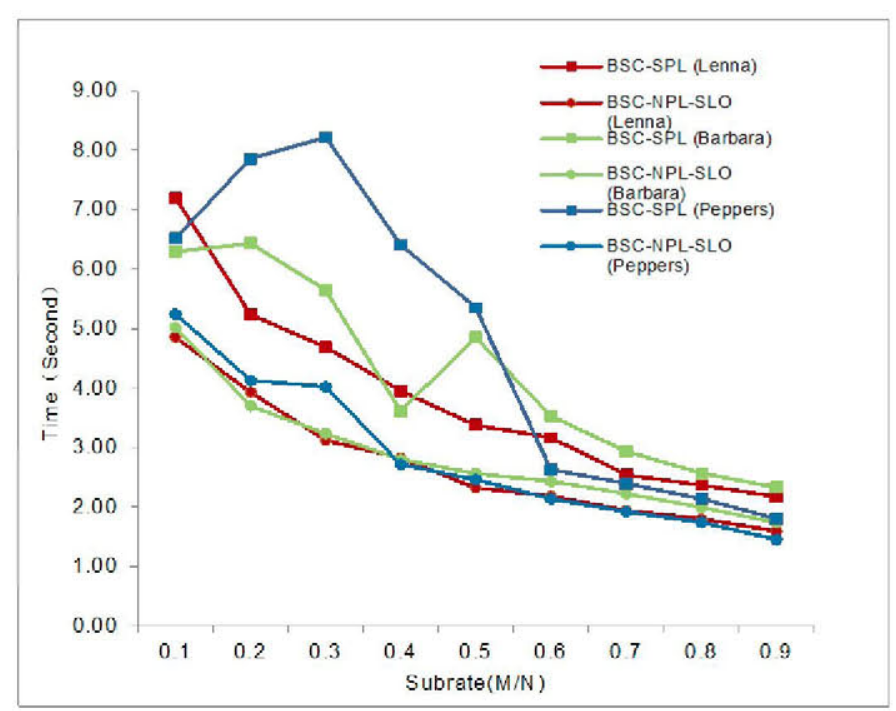

Fig. 2. Recovery performance (time) of the proposed method for block size $32 * 32$ 


\begin{tabular}{|c|c|c|c|c|c|c|c|c|c|c|}
\hline Subrate & $\mathbf{0 . 1}$ & $\mathbf{0 . 2}$ & $\mathbf{0 . 3}$ & $\mathbf{0 . 4}$ & $\mathbf{0 . 5}$ & $\mathbf{0 . 6}$ & $\mathbf{0 . 7}$ & $\mathbf{0 . 8}$ & $\mathbf{0 . 9}$ & Test Image \\
\hline BCS-SPL & 27.664 & 30.445 & 32.472 & 34.200 & 35.774 & 37.347 & 39.123 & 41.266 & 44.630 & Lenna \\
\hline BCS-NPL-SL0 & 27.641 & 30.423 & 32.467 & 34.212 & 35.804 & 37.377 & 39.164 & 41.314 & 44.681 & \\
\hline BCS-SPL & 22.768 & 24.385 & 25.922 & 27.444 & 28.879 & 30.973 & 33.514 & 36.529 & 40.767 & Barbara \\
\hline BCS-NPL-SL0 & 22.783 & 24.440 & 25.971 & 27.538 & 29.224 & 31.081 & 33.504 & 36.566 & 40.813 & \\
\hline BCS-SPL & 27.976 & 28.023 & 29.460 & 32.425 & 33.931 & 36.331 & 37.851 & 39.775 & 42.946 & Pepper \\
\hline BCS-NPL-SL0 & 27.966 & 30.810 & 31.962 & 33.866 & 35.094 & 36.390 & 37.911 & 39.845 & 43.012 & \\
\hline
\end{tabular}

NPL-SL0 are set as follows: $\lambda_{0}$ is set to 6 , Tol is set to 0.00001, max_iterations is set to $200, \sigma$ is set to 0.00005 . We use Gaussian matrix as the measurement matrix.

Quality and computational time results of the recovery process are shown in Table 1 and Fig. 2, respectively. In general, the proposed BCS-NPL-SL0 algorithm has a similar quality performance, but it achieves a significant reduction in computational time (about 30\%).

\section{CONCLUSIONS}

This paper has proposed a faster reconstruction method for block-based compressed sensing images, which first combines a modified Newton method with the SL0 algorithm, and then it applies the BCS-SPL technique. As a result, a faster reconstruction technique is achieved, while keeping the quality similar to the BCS-SPL algorithm.

\section{ACKNOWLEDGMENT}

This work has been partially supported by the Ministerio de Economía y Competitividad of the Spanish Government under projects TEC2010-20412 (Enhanced 3DTV) and TEC201348453 (MR-UHDTV).

\section{REFERENCES}

[1] Candè E J, Wakin M B, "An introduction to compressive sampling," Signal Processing Magazine, IEEE, vol. 25(2), pp. 21-30, 2008.

[2] Gan L, "Block compressed sensing of natural images," Digital Signal Processing, 15th International Conference on. IEEE, pp. 403-406, 2007.

[3] Mun S, Fowler J E, "Block compressed sensing of images using directional transforms," Image Processing (ICIP), 2009 16th IEEE International Conference on. IEEE, pp. 3021-3024, 2009.

[4] Mohimani H, Babaie-Zadeh M, Jutten C, "A fast approach for overcomplete sparse decomposition based on smoothed norm," Signal Processing, IEEE Transactions on, vol. 57(1), pp.289-301, 2009.

[5] Lin Wanjuan, Zhao Rui-zhen, Li Hao, Hu Shaohai, "Reconstruction algorithm for compressive sensing based on smoothed norm and revised newton method," Journal of Computer-Aided Design \& Computer Graphics, vol. 24(4), pp. 478-484, 2012. 